\title{
Functional Outcome of Surgical Management of Tibial Plateau Fractures: A Study in a Tertiary Care Hospital
}

\author{
Md. Nazibullah", ${ }^{1,}$, Md. Tariqul Islam ${ }^{1}$, Tapas Kumer Talukder ${ }^{2}$, A. K. M. Shaharul Islam ${ }^{1}$ \\ ${ }^{1}$ Department of Orthopaedics \& Traumatology, TMSS Medical College, Bogura, Bangladesh \\ ${ }^{2}$ Department of Ortho Surgery, Shaheed Ziaur Rahman Medical College, Bogura, Bangladesh
}

Email address:

dr.nazibullah@gmail.com (Md. Nazibullah)

${ }^{*}$ Corresponding author

To cite this article:

Md. Nazibullah, Md. Tariqul Islam, Tapas Kumer Talukder, A. K. M. Shaharul Islam. Functional Outcome of Surgical Management of Tibial Plateau Fractures: A Study in a Tertiary Care Hospital. American Journal of Biomedical and Life Sciences. Vol. 9, No. 1, 2021, pp. 53-57. doi: 10.11648/j.ajbls.20210901.17

Received: November 8, 2020; Accepted: January 13, 2021; Published: January 30, 2021

\begin{abstract}
Background: Tibial plateau fractures are serious injuries resulting frequently in functional impairment. Low and high-energy tibial plateau fractures usually result from axial loading in combination with valgus stress forces, present a variety of soft tissue and bony injuries that can produce permanent disabilities. Since there are various modalities for fixation of these fractures with satisfactory results, but there is no general consensus as to which modality is the best in terms of functional outcome and proving the superiority of one over the other. Aim of the study: The aim of this study was to evaluate the functional outcome of surgical management of tibial plateau fractures. Methods: It was a prospective observational study and it was conducted in the Department of Orthopaedics \& Traumatology, TMSS Medical College, Bogura, Bangladesh during the period from January 2018 to December 2018. The total of cases included in the study was 56 in number. More than 20 years old patients of both sexes with tibial plateau fractures attended to the mentioned hospital during the first six months of the study were selected as the study population. All simple or Gustilo Anderson Grade I fractures of tibial plateau presenting in patients requiring open or closed reduction and internal fixation to restore the articular anatomy were finalized for this study. Result: In this study among total 56 participants 37 were male which was $66.07 \%$ and 19 were female which was $33.93 \%$. On the other hand in this study we found satisfactory outcome in 50 patients which was $89.29 \%$ and not satisfactory in 6 patients which was $10.71 \%$ among all the participants. Final end result as per Rasmussen's criteria was excellent in $34(60.71 \%)$, good in $9(16.07 \%)$, fair in $7(12.50 \%)$ and poor in $6(10.71 \%)$ patients. Conclusion: In this study we found satisfactory outcome in 50 patients which was $89.29 \%$ and not satisfactory in 6 patients which was $10.71 \%$ among all the participants. These findings may be helpful for the future researchers for any further research in the same issue.
\end{abstract}

Keywords: Tibial Plateau Fractures, Axial, Gustilo Anderson Grade, Schatzker Classification

\section{Introduction}

The tibial plateau is the region of proximal tibia, which comprises of the superior articular surface. It constitutes a major weight bearing area in the body. Its fractures classically were described as bumper or fender's fractures. They gravely affect the biomechanics, stability and range of motion of the knee joint. [1,2] These injuries present with a wide array of fractures, varying from minor hairline cracks with excellent functional outcomes even after conservative treatment to challenging fracture configurations requiring highly experienced surgical hands. The management of these types of injuries has for long been subject of controversies. The spectrum of treatment ranges from simple casting and bracing to skeletal traction and early motion to open reduction and internal fixation. [3] Moreover, the appropriate treatment for injuries of different severities is unclear. A brief review of literature reveals that different avenues are being explored for these fractures. Ali, et al reported a $31 \%$ fixation failure for tibial plateau fracture in their elderly population. [4] Stevens et al noted that only 57\% of cases showed good functional outcome after surgical management of tibial plateau fractures in age $<40$ years. [5] Open reduction and 
internal fixation has a significant complication rate. [6] So, a middle path of minimally invasive technique of closed reduction by ligamentotaxis and stabilizing the fracture by limited internal fixation was developed and practised to overcome the drawbacks of non-operative and operative modalities. [7] These techniques utilize percutaneous screws and Kirschner wires ( $\mathrm{K}$ wires), external fixation frames or combination of external fixation with limited internal fixation. [8] The minimally invasive technique of closed reduction by ligamentotaxis and fixation with percutaneous screws and $\mathrm{K}$ wires, combines attributes to both operative and nonoperative philosophies. The treatment of tibial plateau fractures is continuously evolving. Various techniques have emerged over the last few decades. Until the seventies, these fractures were essentially treated conservatively with a variety of modalities such as traction, cast bracing and spica casting. [9] In the eighties, as the science of internal fixation progressed rapidly, more and more tibial plateau fractures started to get operated. [10] Surgically treated fractures yielded favorable results due to the achievement of better articular congruity, higher stability and early mobilization. As the propensity of high velocity trauma increased, the incidence of complex fractures also increased and thus more and more fractures were subjected to surgical reductions. As more tibial plateau fractures are surgically treated, surgical complications, have become relatively common. Surgical methods for reducing and fixing tibial plateau fractures have evolved over the last three or four decades in order to identify techniques that minimize complications and other techniques that optimize the treatment of complications when they occur. The aim of this study was to evaluate the functional outcome of surgical management of tibial plateau fractures. So all the parts of this study were aimed to the proper objectives of the study.

\section{Objectives}

General Objective:

To evaluate the functional outcome of surgical management of tibial plateau fractures.

Specific Objective:

To assess the mechanism of injuries of tibial plateau fractures.

\section{Methodology \& Materials}

It was a prospective observational study and it was conducted the Department of Orthopaedics \& Traumatology, TMSS Medical College, Bogura, Bangladesh during the period from January 2018 to December 2018. The total of cases included in the study was 56 in number. More than 20 years old patients of both sexes with tibial plateau fractures attended to the mentioned hospital during the first six months of the study were selected as the study population. All simple or Gustilo Anderson Grade I fractures of tibial plateau presenting in patients requiring open or closed reduction and internal fixation to restore the articular anatomy were finalized for this study. Any fracture having Gustilo Anderson severity of more than Grade I, all pathological fractures or fractures having associated condition such as compartment syndrome, ipsilateral meniscal or ligamentous injury, floating knee or any other polytrauma were rejected as they would have affected the rehabilitation and ultimate functional outcome of the study. After recording identification data, antero-posterior and lateral roentgenograms were used to classify the facture according to Schatzker classification. Surgical intervention was done under suitable antibiotic cover and fluoroscopic control and as soon as local soft tissue conditions were favorable, operation was performed. Fracture site reduction was done under fluoroscopic guidance with the use of percutaneous clamps and distracters judiciously and repeat fluoroscopic assessment was done to assess anatomical reduction. Wherever needed open reduction was performed. Buttress plates were used since the proximal end of tibia contains a large amount of cancellous bone and has a tendency of axial deviation or bending under the effect of compression or shearing forces. The different types used were $\mathrm{T}$ shape having a vertical and horizontal limb and helps in preventing a thin cancellous bone from collapsing, L shape having a right or left offset and a double bed to fit into the plateau and Hockeystick plate which is stouter and majority of times used to buttress the lateral plateau. Locking compression plates were used in cases of high energy bicondylar fractures, severe comminution and in osteoporotic bones. Interfragmentary compression cannot be achieved by locked plate alone and therefore, supplementary use of inter-fragmentary screws may be required to prevent loss of reduction and to ensure adequate compression of fragments. For this purpose cortical screws (4.5 $\mathrm{mm}$ of various diameters), cancellous screws (16 mm, $32 \mathrm{~mm}$, partially and fully threaded) and locking screws were used. The implants used were selected according to the fracture configuration and the preference of the surgeon. Post operatively patients were kept on injectable antibiotics for 3 to 5 days and static quadriceps and ankle pump exercises were started from $2^{\text {nd }}$ day. If the fixation was deemed stable then intermittent knee mobilization was started once pain subsided. Weight bearing was deferred until evidence of union was seen on $\mathrm{X}$ ray. Partial weight bearing was started at around 10 to 14 weeks depending upon the fracture configuration. The results of this study were evaluated using the functional grading of Rasmussen et al. [11]

\section{Result}

In this study among total 56 participants 37 were male which was $66.07 \%$ and 19 were female which $33.93 \%$ was. Among total population highest $30.36 \%(n=17)$ were from $31-40$ years' age group. Then $25 \%(n=14)$ were from $41-50$ years' age group, $23.21 \%(\mathrm{n}=13)$ were from $20-30$ years' age group, $14.29 \%(\mathrm{n}=8)$ were from $51-60$ years' age group and only $7.14 \% \quad(n=4)$ were from $>60$ years' age group. In analyzing the mechanism of injuries of the participants we 
found, the highest $27 \%$ injuries occurred by road traffic accidents. Then in $23 \%$ cases the mechanism was low energy, in $18 \%$ cases it was high energy, in $16 \%$ cases it was simple falls, in $12 \%$ cases it was sports and in only $4 \%$ cases it was fall from height. The average duration of hospitalization of the participants was 7.5 days (range 4-8 days). In total 36 patients were operated within 2 to 3 days of injury and showed excellent to good results. Twelve patients presented at 5-7 days of injury. Amongst them, those patients who had swelling around the proximal leg were kept on skeletal traction and were operated as soon as local tissue condition was optimized for surgery. In total 9 patients had delayed presentation and were operated as soon as next $\mathrm{O}$. $\mathrm{T}$ was available. One patient presented near about 2 months post injury and had remained on bed rest since then. The distribution of patients according to Schatzker classification and their outcome of treatment are summarized. In total 6 patients were managed with cancellous screws; 15 patients underwent open reduction and fixation with plating while plating supplemented with bone grafting was done in ten patients. Plating with MIPO technique was performed in 11 patients and external fixator was applied in 3 patients. In total 8 patients had fixation done with dual plating. Average time gap between operation and partial weight bearing was around 8.75 weeks ( 8 to 13 weeks). The mean period of radiological union was 12.75 weeks (range 12 to 16 weeks). Most of the patients were allowed complete weight bearing at 11 to 14 weeks. Average time gap for complete weight bearing was 13.25 weeks. Most of the patients $(n=34)$ had $120^{\circ}$ or more knee flexion. Average range of motion was $112.8^{\circ}$. The distribution of patients on the basis of time to union is summarized. The cause of pain could not be sorted although they could perform all activities of daily living. One patient had infection on 7 th post-operative day and was managed with intravenous antibiotics and subsequently developed implant exposure and implant removal was done followed with skin grafting for soft tissue coverage and kept immobilized. It belonged to Schatzker type VI and remained in non-union at follow up period. Another patient had infection which was managed with antibiotics and debridement although ultimate outcome was not acceptable. One patient managed with dual plating had R. O. M of $60^{\circ}$ by the end of the year. This patient after implant removal was started on physiotherapy and finally had R. O. M of $90^{\circ}$. One patient had varus deformity of $10^{\circ}$ due to collapse of medial condyle, probably due to early mobilization. In this study we found satisfactory outcome in 50 patients which was $89.29 \%$ and not satisfactory in 6 patients which was $10.71 \%$ among all the participants. Final end result as per Rasmussen's criteria was excellent in $34(60.71 \%)$, good in $9(16.07 \%)$, fair in $7(12.50 \%)$ and poor in $6(10.71 \%)$ patients.

Table 1. Age distribution of participants $(N=56)$.

\begin{tabular}{lll}
\hline Age (Yrs.) & $\mathbf{n}$ & \% \\
\hline $20-30$ & 13 & 23.21 \\
$31-40$ & 17 & 30.36 \\
$41-50$ & 14 & 25.00 \\
$51-60$ & 8 & 14.29 \\
$>60$ & 4 & 7.14 \\
\hline
\end{tabular}

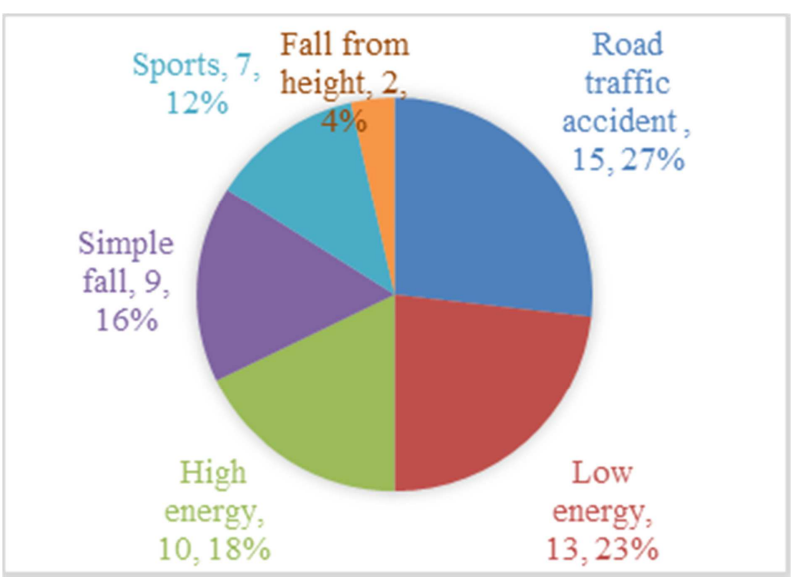

Figure 1. Distribution of mechanism of injuries $(N=57)$.

Table 2. Distribution of patients according to Schatzker classification and their outcome $(N=56)$.

\begin{tabular}{|c|c|c|c|c|}
\hline \multirow{2}{*}{ Schatzker type } & \multirow{2}{*}{ Total patients $(n=56)$} & \multicolumn{2}{|c|}{ Patients got acceptable outcome } & \multirow{2}{*}{$\begin{array}{l}\text { Patients without acceptable } \\
\text { outcome }\end{array}$} \\
\hline & & $\mathbf{n}$ & $\%$ & \\
\hline I & 6 & 6 & 100 & \\
\hline II & 12 & 12 & 100 & \\
\hline III & 5 & 4 & 80 & \\
\hline IV & 8 & 6 & 75 & $6(10.71 \%)$ \\
\hline $\mathrm{V}$ & 14 & 13 & 92.86 & \\
\hline VI & 11 & 9 & 81.82 & \\
\hline Total & 56 & 50 & 89.29 & \\
\hline
\end{tabular}

Table 3. Distribution of patients on basis of time to union $(N=56)$.

\begin{tabular}{lll}
\hline Union & n & \% \\
\hline Less than 12 weeks & 31 & 55.36 \\
12 to 14 weeks & 9 & 16.07 \\
More than 14 weeks & 15 & 26.79 \\
Non union & 1 & 1.79 \\
Total & 56 & 100 \\
\hline
\end{tabular}

Table 4. General grading of patient's outcome $(N=56)$.

\begin{tabular}{lll}
\hline Grade & $\mathbf{n}$ & $\mathbf{\%}$ \\
\hline excellent & 34 & 60.71 \\
good & 9 & 16.07 \\
fair & 7 & 12.50 \\
poor & 6 & 10.71 \\
\hline
\end{tabular}




\section{Discussion}

Generally tibial plateau fractures, one of the commonest intra articular fractures, are major traumatic injury occurring due to road traffic accidents, fall from height, violence etc. It is sometimes associated with other bony or soft tissue injuries. Any fracture around the joint (especially weight bearing joint in the lower limb) is of paramount importance as it would result in significant morbidity and quality of life. Hence the treatment of upper tibial fractures with intra articular extension has become a challenge for orthopaedic surgeons. Keeping this aim at high, we presented the clinical study of surgical treatment of 56 closed tibial plateau fractures in this study. The analysis of the results were made in terms of age, sex distribution, mode of violence, analysis of the type and the functional outcome. We have presented the various types of tibial plateau fractures in our Indian setup. It is found that, the zeal of modernization, mechanization and industrial development made more automobile accidents due to increase in population and automobiles. In our study we observed the majority of fractures occur between 20 to 50 years of age with maximum incidence involving productive age group of 31 to 40 years $(30.36 \%)$. The mean age in this study was 42.76 years. In a similar study done by Rasmussen et al the average age of patients was 55 years.[11] In our study the majority of patients were male $(66.07 \%)$. This can be attributed to our Indian set up where the female population largely remains indoors and is less prone to automobile accidents. In this study the commonest mode of injury was road traffic accident $(27 \%)$. This did not correlate well with previous study by Chiax et al who in their series reported that $71 \%$ of the injuries occurred due to RTA. [12] There was a significant preponderance to the right side in laterality of the fracture $(57 \%)$. In this series we studied 56 cases of simple tibial plateau fractures treated only by surgical method. Different authors use different criteria for surgical management of these fractures. Seppo E Honkoenen in his series of 130 tibial plateau fractures, conducted surgery taking into consideration condylar widening of $>5 \mathrm{~mm}$ and lateral condyle step off $>3 \mathrm{~mm}$. [13] The indication for surgery in these types of injuries has evolved steadily with time. Burri, et al in his study in 1979 advised internal fixation at $1 \mathrm{~mm}$ of depression, Hohl et al and Segal et al advocated fixation at $5 \mathrm{~mm}$ of depression and Honkonen et al took 3 $\mathrm{mm}$ of depression in consideration in his study in 1993. [14, 15] In this study the indication for surgery were the same standard indications as for those tibial plateau fractures, 3 $\mathrm{mm}$ depression was considered as an indication for surgery in this series. In this study we found satisfactory outcome in 50 patients which was $89.29 \%$ and not satisfactory in 6 patients which was $10.71 \%$ among all the participants. The period of immobilization was individualized depending upon the rigidity of fixation. The benefits of early knee movement include reduced knee stiffness and improved cartilage regeneration. However these benefits are to be cautiously weighted against their negative impact such as loss of fracture reduction, failure of internal fixation and compromised soft tissue healing. J Schatzker and Robert Mcbroom $^{5}$ stated that the prognosis is given by the degree of displacement, type of fracture, method of treatment and quality of post-operative care. The problem faced during this study was infection and wound dehiscence in 6 patients ultimately leading to unacceptable outcome. The infection might be attributed to nosocomial infection. In spite of all the complications we were able to achieve $60.71 \%$ excellent, $16.07 \%$ good results. In total $10.71 \%$ of patients had unacceptable outcomes. These results are at par with other documented studies. Ebraheim et al in his series of 117 tibial plateau fractures had excellent results in $68 \%$ of cases, good in $13 \%$, fair in $11 \%$ and poor in $8 \%$ of the patients.[16] Final end result of our study as per Rasmussen's criteria was excellent in $34(60.71 \%)$, good in $9(16.07 \%)$, fair in 7 $(12.50 \%)$ and poor in $6(10.71 \%)$ patients which was similar to many other international studies.

\section{Limitations of the Study}

This was a single centered study with a small sized sample. So the findings of this study may not reflect the exact scenario of the whole country.

\section{Conclusion and Recommendations}

In this study we found satisfactory outcome in 50 patients which was $89.29 \%$ and not satisfactory in 6 patients which was $10.71 \%$ among all the participants. These findings may be helpful for the future researchers for any further research in the same issue. For getting more specific findings we would like to recommend for conducting more studies regarding the same issue with larger sized sample.

\section{Funding Source}

Self.

\section{Conflict of Interest}

None Declared.

\section{Approval}

Got Approval from respective department.

\section{References}

[1] Leadbetter GW, Hand FM. Fractures of the tibial plateau. J Bone Joint Surg. 1940; 22 (3): 559-68.

[2] Barr JS. The treatment of fracture of the EXTERNAL tibial condyle: (Bumper fracture). J American Med Assoc. 1940; 115 (20): 1683-7.

[3] Apley AG. Fractures of tibial plateau. Clin Orthop North Am. 1979; 10: 61-74. 
[4] Ali AM, El-Shafie M, Willet KM. Failure of fixation of tibial plateau fractures. J Orthop Trauma. 2002; 16 (5): 323-9.

[5] Stevens DG, Beharry R, McKee MD, Waddell JP, Schemitsch EH. The long-term functional outcome of operatively treated tibial plateau fractures. J Orthop Trauma. 2001; 15 (5): 312-20.

[6] Schatzer J, McBroom R, Bruce D. The tibial plateau fracture: the Toronto experience 1968-1975. Clin Orthop. 1979; 138: 94-104.

[7] Keogh P, Kelly C, Cashman WF, McGuinness AJ, O'Rourke SK. Percutaneous screw fixation of tibial plateau fractures. Injury. 1992; 23 (6): 387-9.

[8] Mikulak SA, Gold SM, Zinar DM. Small wire external fixation of high energy tibial plateau fractures. Clin Orthop Relat Res. 1988; 356: 230-8.

[9] Fyshe TG. Fractures of tibial condyles. Canadian Med Assoc J. 1952; 67 (2): 103.

[10] Savoie FH, Vander Griend RA, Ward EF, Hughes JL. Tibial plateau fractures. A review of operative treatment using AO technique. Orthoped. 1987; 10 (5): 745-50.
[11] Rasmussen PS. Tibial condyle fractures. Impairment of knee joint stability and indication for surgical intervention. J Bone Joint Surg Am. 1973; 55 (7): 1331-50.

[12] De Mourgues G, Chiax D. Treatment of tibial plateau fractures. Rev Chir orthop Reparatrice Mot. 1969; 55 (6): 575-6.

[13] Honkonen SE. Indications for surgical treatment of tibial condyle fractures. Clin Orthop Relat Res. 1994; 302: 199-205.

[14] Burri C, Bartzke G, Coldewey J, Mugglar E. Fractures of tibial plateau. Clin Orthop Relat Res. 1979; 138: 84-93.

[15] Bowes DN, Hohl M. Tibial condyle fractures. Evaluation of treatment and outcome. Clin Orthop Relat Res. 1982; 171: 104-8.

[16] Ebraheim NA, Sabry FF, Haman SP. Open reduction and internal fixation of 117 tibial plateau fractures. Ortho Blue Journal. 2004; 27 (12): 1281-7. 